\title{
LEVELLING REFRACTION RESEARCH, ITS PRESENT STATE AND FUTURE POSSIBILITIES
}

\section{T.J.Kukkamäki}

Finnish Geodetic Institute, Helsinki

When I had to start the Second Levelling of Finland, 43 years ago, after the first observing summer I made one important and happy finding. The vibration of the image in the levelling instrument made the levelling difficult in middle summer while in spring and autumn this trouble was not so bad. So I proposed that levelling work must be interrupted for the six weeks from the midsummer to the beginning of $\mathrm{Au}-$ gust and that was accepted. The fact that just these weeks are the best vacation time in Finland made our colleagues in the institute jealous, but it was not our fault, and we were happy.

Then I made one other finding which turned out to be rather stupid. The vibration of the rod image in levelling instrument's view was bad at noon but was the better the farther from the noon the observation was made. Even when the sun was below the horizon this image seemed to be clear and unmovable. So I proposed that we should construct fitting instrumentation for observations in dark period, i.e. some devices for lighting the rod scale and the levelling instrument itself. Before starting the practical measures in that direction, we made a lot of research into the microclimatic and also optical conditions in the first three meters of atmosphere above the ground, and I think, we got rather clear picture of the conditions there. As these details seem to be unclear to many scientists, even to those who have published about the levelling refraction, I should like tell something about our experience on the matter.

We knew from the numerous meteorological publications the general variation of the temperature in the three lowest meters of air. In night, because of Austrahlung, the ground is colder than the air just above it. Thus the temperature of the air increase with the height, at least up to three meters; the temperature gradient is positive. Soon after the sunrise the Einstrahlung surpasses the Austrahlung, and the temperature of the air is decreasing with the height; the vertical temperature gradient is negative. The absolute value of the gradient

$$
293
$$

E. Tengström and $(G$. Teleki (eds.). Refractional Influences in A strometry and Geodesy, 293-299. Copyright $\odot 1979$ by the IAU. 
increases with the morning hours up to 13-15 hours in the afternoon. Since that the absolute value of the gradient decreases and reaches zero about at the sunset. In course of the time when the sun is below the horizon the positive gradient increases lowly until the rising sun turns the vertical temperature gradient to zero and to the negative values.

The absolute values of the vertical gradient are the greater the clearer the sky is on day or night.

According to the information given in the microclimatological text-books with the negative gradient the warmer, i.e. lighter, air near the ground is rising turbulently. It means that air bubbles, some meters large and differing about $1^{\circ} \mathrm{C}$ from the surrounding air, are rising in the lowest air layers. This upwards moving in bubbles makes the vibration of the rod scale and one can compute with some theoretical assumptions that the vibration increases with the 1.5 power of the sight length and is for a sight lenght of 50 meter in average conditions about $1 \mathrm{~mm}$. The amplitude depends strongly on the vertical gradient.

In order to verify this reasoning, we made observations series with sight lengths $25,50,75,100,125$ and $150 \mathrm{~m}$ around the whole 24 hours. We got the exponent 1.68 instead of the theoretical 1.5 and the amplitude was $1.2 \mathrm{~mm}$ for $75 \mathrm{~m}$ sight length. The frequency of the vibration was for small gradient one in second and with great gradient $5-10$ in a second.

Our observations agreed the theoretical values satisfactorily. The vibration allows relatively safe pointing to the center of the vibrating scale image unless the vibration is not too large. Strong vibration force the observer to shorten the sight length and further to stnp the observation. It was proved that our decision to keep summer vacation of levelling observers just in the finest summer weather period was scientifically grounded.

But now, how it is with the night observations. In the night the vertical gradient is positive, i.e. the colder and heavier air layers are under the lighter ones and no turbulence appears. Under the open sky, however, a wind of some decimeters in second always appears. This causes a slow swaying of the air layers in periods on tens of seconds or even minutes. The image of rod scale under these conditions is moving up and down in the same slow periodes. The image looks sharp and beautiful and not moving, but when you make a new pointing after tens of seconds or minutes the reading might deviate from the earlier considerably.

In order to get some idea of the amount this movement we continued the above mentioned vibration observations in night time also. We found that the amplitude of the swaying increases with the second power of the sight length. The amplitude was with a temperature gradient of $+0.2 / 1 \mathrm{~m}$ in average $1 \mathrm{~mm}$ for $75 \mathrm{~m}$ sight length. 
So this result showed that the levelling in night, when the vertical gradient always is positive, is not possible with an accuracy requested for precise levelling.

The vibration is symmetrical in vertical and horizontal directions. It is strongly depending on the gradient and consequently on cloudiness. That cannot cause any systematical error. That only limits observing possibilities and makes the accidental error larger. Normal sight length in precise levelling is 40,50 or $60 \mathrm{~m}$. I cannot understand that e.g. in USA even $150 \mathrm{~m}$ sight length was accepted still in the instructions of 1929. When vibration makes observing difficult the sight length is to be shortened. When even $30 \mathrm{~m}$ is not short enough the observation is to be interrupted. I think that this understanding about the vibration is accepted generally.

The understanding of the slow swaying is not so selfevident, but fortunately our colleagues have not been so stupid as I was in the beginning of my work that had tried to observe when the gradient is positive.

Naturally the gradient is greater nearer to the ground. So the horizontal sight in levelling bends more in uphill sight where it comes nearer to the ground than in the downhill sight. In order to get some picture of this phenomenon and possibly to derive some quantitative correction we studied the text-books and all possible other sources. We found names like Lallemand, Hugershoff, Kohlmüller, de Graaf-Hunter, Cole and Bomford. All these and many others had made researches into the levelling refraction. They based their computation on different functions for temperature as

$$
\begin{aligned}
& t=a+b \cdot \log (z+c) \\
& t=a+b \cdot z^{2} \\
& t=a+b \cdot z+c \cdot z^{2}
\end{aligned}
$$

Most of these scientists were satisfied with qualitive results. Rather few direct observations were carried out about the temperature variation with the height in different hours of day and night.

Further we have noticed that in some cases rather strange apprehensions came in view. For instance one serious scientist said in discussion with me 40 years ago, that light is bending in morning upwards as the temperature is rising and in afternoon it is bending downwards as the temperature is going down. In an article of Bulletin géodésique 20 years ago it is said that soon after the noon when the turbulent mixing reaches its maximum the temperature gradient is zero and no bending of light beam appears.

In the state of this kind of uncertainty we decided to make our own observations of the temperature in air up to three meters and simultaneously to try to observe any possible deviations in levelled height differences. 
At first we observed with thermocouple the temperature at $1 / 3,1$ and 3 meter, but we found soon that the second derivative of the temperature is not possible to determine from single set of temperature readings. One needs long recordings. Since that we have observed only one temperature difference between 0.5 and 2.5 meter. The temperature function

$$
t=a+b \cdot z^{c}
$$

is used. The linear factor in it is computed from the direct observation but the exponent $c$ is computed from the long temperature recordings made by Best in South England. I derived the exponent $c$ for different hours, different months and different latitudes. This is, however, too sophisticated, and my colleague Professor Hytönen has shown that one can use the average value $c=-0.1$ in all circumstances without causing any significant error.

In order to see the refraction effect in levelling as clearly as possible we thought it is better to use an extraordinary sight length e.g. 100 meter as then the effect should be four times larger than with 50 meter, and the effect is not covered by the other observing errors. So we levelled a slope $2 \mathrm{~km}$ long with 18 meter elevation difference in all hours of day and night, with an exception of period from 22 to 03 . From the graph we see that the observed deviations agree the values computed from the simultaneous gradient observations beautifully. The deviations reached values over $10 \mathrm{~mm}$ with 100 meter sight length and $4 \mathrm{~mm}$ with the regular 50 meter sight length.

As the gradient observations and the used levelling refraction formulas gave such a consistent result, we concluded that though other errors cover the refraction effect at a single instrument station, it must give a result which has at least a correct direction on longer observation series as at one bench mark interval:

As further the observing of the temperature gradient with a resistance thermometer was easy causing no waisting of time or needing no additional personnel, since 1937 we have observed the gradient at each instrument station and computed levelling refraction correction for each instrument station and applied this correction in the reduction of the whole Second Levelling of Finland. In average this correction is $0.06 \mathrm{~mm}$ per $1 \mathrm{~m}$ height difference, i.e. we get a hill, $1000 \mathrm{~m} \mathrm{high}$, because of systematic levelling refraction $60 \mathrm{~mm}$ too low.

Here I have described our efforts to determine and to correct the levelling refraction in Finland. In the years after the Second World War there have been several researches into the levelling refraction and I should like to mention here the names Brocks, Reissmann, Hase, Balasz, Entin, Strusinsky and Kneiss1, who all have studied this interesting question. Yesterday we heard already important reports on theoretical or practical results and today shall hear more. 


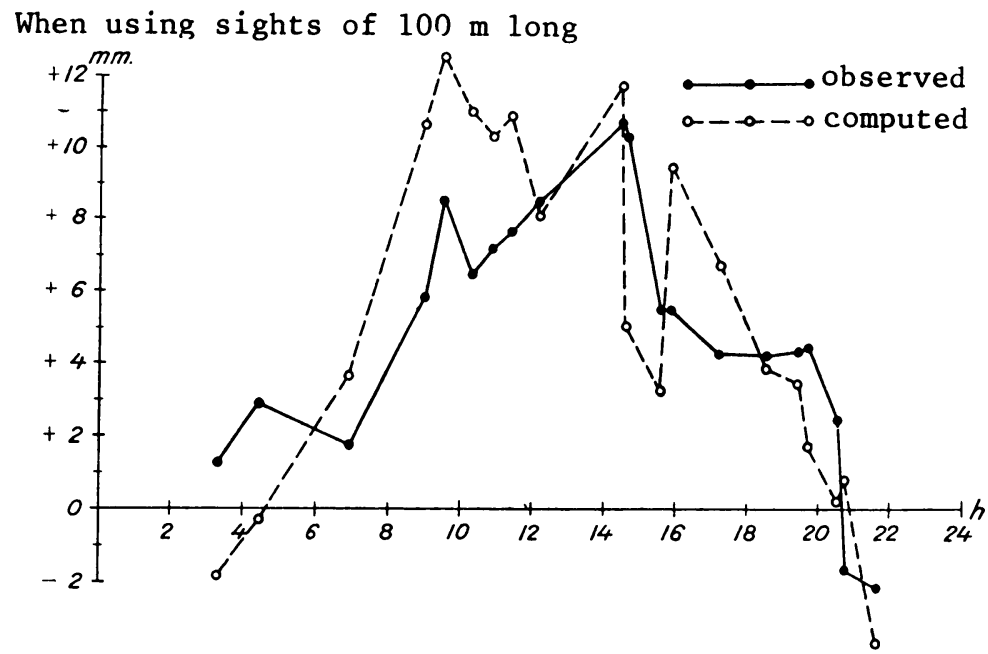

When using sights of $50 \mathrm{~m}$ long

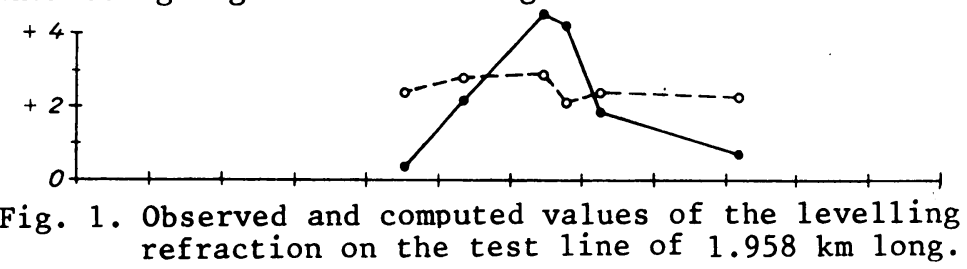

The purpose of this presentation of mine is to suggest some you will study the levelling refraction still more and more, as $I$ think that this phenomenon is not yet unravelled satisfactorily. You may use classical methods but it would be still better to use electronics and electronic computors which have yielded so many magical solutions nowadays.

\section{DISCUSSION}

B. Garfinkel: I have two questions to ask. I was under the impression that in the Kukkamäki formula for the temperature profile, there are two important parameters, called $b$ and $c . c$ is the exponent. And furthermore the exponent was. expected to be affected by the time of observation, particularly the time of the day, and also the season of the year. Now today you said that one can choose $c$ as a fixed constant, something like -0.1 . Now how do you reconsile these two opinions?

T.J. Kukkamäki: It is so: the first opinion was the old opinion of a young man, then this second one is the new opinion of an old man. I 
think that the last one is wiser than the first one.

B. Garfinkel: I am very pleased, it is now much simpler to make the calculations. I have, however, a second question. Suppose you want to measure these parameters $b$ and $c$ in the field at a particular time of observation. Could you use three temperature measurements at different heights, obtaining two differences and then from those differences calculate the two parameters $b$ and $c$ at the actual time of observation? Would that be a good plan?

T.J. Kukkamäki: We can do it, but we cannot get any dependable result from that. We cannot get $c$ from those two differences, as it is changing so much that there is no sense to compute it. Already in the beginning of our work we saw this, and therefore we used recordings of Best and we determined the exponent $c$ statistically. Later we saw that even that was not necessary, but we can use an average value for $c$.

B. Garfinkel: What do you do about the parameter b? Is that also a fixed number?

T.J. Kukkamäki: The parameter $\mathrm{b}$ must be determined along the measurements. After some minutes we will hear the presentation of Holdahl about a method to compute $b$ for old measurements, where no gradient thermometers are used, and only information about cloudiness and direction of the slope is available. When you are making new levellings you have the possibility to make these direct measurements, please do that. It is easy, simple and not at all expensive.

E. Tengström: Professor Kukkamäki knows that there are plans going on to make a connection between the precise levelling of Finland and the corresponding of Sweden. And, of course, we have to cross the Gulf of Bothnia. You have, as I understand, done levelling through islands up to the lighthouse Märket. And we have carried out our levelling on to the lighthouse Understen. When I talked to Kakkuri about your levelling across the Finnish islands, he told me that this type of topography, that is precise levelling over water surfaces, is extremely nice to make, with very small errors in summertime. And he observed, that during this time it was good to be as near the surface of the water as possible, in order to have the turbulence effects not so big, because of the constancy of the temperature of the water and air. Now, you know we are talking about the two-colour method. The rest, that is the distance Understen on to Märket, cannot be hydrostatically levelled because of the ships.

\section{T.J. Kukkamäki: And because of the lack of money!}

E. Tengström: OK, can be. So we thought that the first thing we should do, after these experiments along our testbase in Uppsala, was to try trigonometrical levelling and correction for refraction over the remaining distance mentioned. So if we have our instruments rather near the surface of the water, which means much better conditions than we 
have here at the testbase topography, what accuracy shall we aim at in order to make a positive contribution to the connection between our two levelling nets? If the conditions are better than here, perhaps we may not be pessimistic in hoping to get an accuracy of $0 " .5$.

T.J. Kukkamäki: I can tell, that when we measured from the Finnish mainland to lighthouse Märket, we made about 100 watercrossings, and the accuracy was $\pm 25 \mathrm{~mm}$. So to make a significant contribution to the connection Märket - Understen, the accuracy must be something like $\pm 25 \mathrm{~mm}$, not essentially worse. The accuracy of a levelling around the Gulf of Bothnia is of that magnitude, also.

E. Tengström: The distance between the islands of Understen and Märket is $12 \mathrm{~km}$. So I believe then it will be possible.

L. Hradilek: We are performing a spirit levelling in the valley of the High Tatras, with the elevation difference $700 \mathrm{~m}$ over $15 \mathrm{~km}$ distance. What accuracy is attainable when doing our best and applying all recent improvements?

T.J. Kukkamäki: You are making very careful work in Czechoslovakia, and your random precision must be high. But if the roads are gently sloping, and you are able to use $50 \mathrm{~m}$ sightlengths, the systematic levelling refraction can be rather dangerous. In our country, where slopes are very gentle, the systematic error caused by refraction is $0.06 \mathrm{~mm}$ per $1 \mathrm{~m}$ elevation difference on $1000 \mathrm{~m}$ high mountains a $60 \mathrm{~mm}$ levelling refraction is possible. So you can imagine what is the magnitude of that correction. We may expect a real accuracy of some centimeters. 